\section{Cahiers de Narratologie}

Analyse et théorie narratives

11 | 2004

Figures de la lecture et du lecteur

\title{
Colette et son lecteur
}

Une stratégie du déplacement

Marie-Odile André

\section{(2) OpenEdition}

Journals

Édition électronique

URL : http://journals.openedition.org/narratologie/12

DOI : 10.4000/narratologie.12

ISSN : 1765-307X

Éditeur

LIRCES

Référence électronique

Marie-Odile André, "Colette et son lecteur », Cahiers de Narratologie [En ligne], 11 | 2004, mis en ligne le 01 janvier 2004, consulté le 19 avril 2019. URL : http://journals.openedition.org/narratologie/12 ; DOI : 10.4000/narratologie. 12

Ce document a été généré automatiquement le 19 avril 2019

\section{(c) $($ i) $(\Theta)$}

Cahiers de Narratologie - Analyse et théorie narratives est mis à disposition selon les termes de la licence Creative Commons Attribution - Pas d'Utilisation Commerciale - Pas de Modification 4.0 International. 


\title{
Colette et son lecteur
}

\author{
Une stratégie du déplacement
}

\author{
Marie-Odile André
}

1 L'approche que nous souhaitons proposer des rapports entre Colette et son lecteur est délibérément orientée selon une perspective qui vise à analyser ce qui se joue là du point de vue de la trajectoire de l'écrivain et de sa progressive légitimation ${ }^{1}$. Dans cette perspective, les textes apparaissent, en effet, comme les instruments d'une conquête progressive de légitimité, elle-même lisible à travers une série de données textuelles spécifiques parmi lesquelles figurent au premier chef les relations qui se construisent entre Colette et son lecteur ${ }^{2}$. Centrées, comme on va le voir, sur une démarche de renégociation, au sein même des oeuvres, du pacte de lecture, ces relations ont pour enjeu une modification du mode de lecture des textes qui a, elle-même, pour effet la construction progressive d'une autre position narrative et d'une autre image de l'écrivain.

2 Trois œuvres des années 20-30 - La Naissance du jour , « Gribiche » et « La Lune de pluie » - constitueront notre corpus : leurs dates respectives de publication qui correspondent à la période où se joue puis s'établit la consécration définitive de Colette ainsi que le statut générique original qui leur est commun justifient le rapprochement ici opéré. Quant à l'analyse des modalités particulières selon lesquelles s'actualise pour chacun d'eux la relation avec le lecteur, elle permettra de cerner les variantes et les évolutions d'une stratégie récurrente dans l'écriture colettienne.

Formes et enjeux de la présence du narrataire dans les textes

Quelques repères minimaux s'imposent concernant les traces scripturales du lecteur dans les textes colettiens, tant en ce qui concerne les formes qu'elles peuvent prendre que le contexte générique qui leur donne sens.

4 Les traces d'un narrataire sont présentes chez Colette, de manière plus ou moins accentuée selon les textes, sous la forme d'un narrataire extradiégétique, peu ou prou identifiable comme lecteur, auquel s'adresse l'instance énonciatrice. On y retrouve ainsi ces signaux bien connus ${ }^{5}$ que sont l'interpellation directe du lecteur, la présence d'un "vous", l'utilisation assez fréquente d'un «nous» ou d'un "on», des questions ou pseudo-questions, des rectifications, etc. 
5 Mais pour être interprétée de façon pertinente, cette présence d'un narrataire invoqué doit être mise en relation avec deux données importantes de l'écriture colettienne: le statut particulier de l'instance narratrice et la position d'autorité qui est volontiers la sienne.

6 On sait que Colette a toujours joué avec les genres de l'écriture du moi, depuis le pseudojournal de Claudine jusqu'au recueil de souvenirs (La Maison de Claudine ou Sido) en passant par le roman à la première personne (La Vagabonde). On sait aussi que la lecture autobiographique de ses textes constitue une donnée essentielle de sa réception de sorte que le jeu entre autobiographie et fiction constitue un élément central de sa démarche d'écriture.

7 Les textes comme La Naissance du jour, «Gribiche» ou «La Lune de pluie », ont pour particularité commune (inaugurée par le premier) de mettre en scène, dans un cadre fictionnel sur lequel nous reviendrons, un personnage qui est aussi le narrateur et qui se donne également pour l'écrivain qu'est Colette. On se retrouve donc dans une configuration ayant toutes les apparences de l'autobiographie, où le personnage, le narrateur et l'auteur semblent se confondre d'autant que le texte délivre suffisamment d'éléments ou d'allusions biographiques pour que le lecteur soit sans cesse invité à superposer les figures de la narratrice et de Colette et donc à lire les adresses qui lui sont faites à travers le narrataire comme des adresses de l'auteur au lecteur effectif de l'oeuvre. D'où cette impression d'une proximité dialogique que produit la lecture des textes de Colette et d'une liaison forte qui s'établit entre Colette et son lecteur. Le phénomène est d'autant plus accentué que, dans le dispositif décrit, le «je» étant personnage, narratrice intra et homo-diégétique, mais aussi auteur, il a toutes les prérogatives de ce dernier, explicitant, au sein même des œuvres, à travers un discours métatextuel, ses intentions et son travail de composition, resituant ses textes par rapport aux productions antérieures et s'employant à en orienter la lecture à venir.

8 A cela s'ajoute le fait que les textes colettiens se caractérisent par une forte autorité exercée par la narratrice sur son texte et sur son lecteur: maîtrise du récit à travers la part d'information livrée ou refusée, tendance à la généralisation et aux jugements de valeur qui ont pour effet d'" enrôler» le lecteur (à travers, par exemple, l'usage de présents de vérité générales, de démonstratifs à forte portée idéologique, de comparaisons) et de le placer sous sa dépendance, y compris quand il s'agit de manier le paradoxe pour retourner certaines idées reçues.

La Naissance du jour ou la renégociation d'un pacte de lecture

9 La relation qui s'établit entre Colette et son lecteur dans La Naissance du jour est particulièrement significative à analyser dans la mesure où elle est indissociable d'un enjeu d'ordre métatextuel portant sur le statut générique de l'oeuvre et que se joue, au sein même du texte, la renégociation de son pacte de lecture.

10 Cet enjeu d'un texte qui met en scène la question de sa lecture est explicité d'emblée à travers les deux épigraphes qui ont successivement accompagné le texte (jouant parfaitement ce rôle de commentaire par anticipation dont parle G. Genette ${ }^{6}$ ). La première - «ce "je" qui est moi et qui n'est peut-être pas moi »-, présente dans la prépublication en revue et censée correspondre à une formule de Proust, met l'accent sur le caractère problématique de la ressemblance autobiographique. La seconde, présente dans l'édition définitive, reprend cette même thématique dans une formule qui, s'adressant directement au lecteur, le suppose susceptible d'une erreur de lecture: 
«Imaginez-vous à me lire, que je fais mon portrait? Patience : c'est seulement mon modèle.» Or cette seconde épigraphe est, rappelons-le, la reformulation, sur un mode plus direct, d'une formule elle-même présente dans le texte : «Imagine-t-on, à me lire, que je fais mon portrait? Patience : c'est seulement mon modèle. » Le passage du « on » au « vous » et la mise en exergue épigraphique de la formule place, en fait, La Naissance du jour sous le signe de cette interrogation au lecteur et de la rectification qui la suit, rectification dont l'enjeu est bien la question de l'identité problématique à soi du sujet écrivant.

11 Une telle démarche ne va pas sans paradoxe, au moins apparent, puisque l'ouvrage met en scène un personnage d'écrivain que tout - même son nom - invite à identifier à Colette, de sorte que le texte semble jouer avec une dimension autobiographique qu'il s'emploie, à la fois, à construire et à détruire. En réalité, le texte établit une relation ambiguë et complexe avec son lecteur : d'un côté, il fournit les éléments qui autorisent ou confortent cette lecture autobiographique; d'un autre, il la conteste à travers une série de mises en garde directes ou indirectes visant elles-mêmes à instaurer un régime de lecture qui, lié au caractère indécidable du statut générique du texte, échappe à la lecture autobiographique auxquels ses textes antérieurs ont été soumis.

12 La lecture autobiographique habituellement faite des textes de Colette se trouve, en apparence, confortée par une confusion entre personnage, narrateur et auteur qui s'étaye des multiples allusions et références à la vie ou à l'oeuvre de l'écrivain ${ }^{7}$ : Dans ces conditions, le lecteur supposé est un lecteur familier de l'oeuvre, un lecteur fidèle, à qui le texte semble donner les moyens de se reconnaître dans un univers largement balisé.

Cependant les relations de la narratrice-auteur avec son lecteur ne se bornent pas à cette confortable et fallacieuse familiarité. Ce lecteur, on l'a vu avec l'épigraphe, est supposé susceptible de commettre des erreurs de lecture; il apparait comme un lecteur suspect aux yeux de la narratrice-auteur (et davantage, sans doute, aux yeux de l'auteur que de la narratrice, du fait du caractère paratextuel de l'épigraphe).

14 Cette suspicion s'exprime dans le texte à travers une réaction que la narratrice lui prête pour mieux la désigner aussitôt comme inadéquate et inadaptée :

«Que faire de cette épave? Quel embarras... Et puis, pensez-vous, la gêne de nos âges respectifs, de la différence d'âge? Que vous êtes loin de ce qui arrive en pareil cas... » (334)

15 A quoi, elle ajoute ce commentaire, qui ne laisse guère de doutes sur la façon dont elle anticipe sur ce que sera fatalement la mauvaise lecture d'un lecteur coupable à la fois de préjugés et d'un usage stéréotypé du langage :

«Non, non, quand j'écris "quel embarras", je ne veux pas qu'un lecteur plus tard,

s'y trompe ».

16 Le texte va plus loin, qui construit, à travers le narrataire invoqué, une série de figures de «mauvais lecteurs » plus explicitement désignés et spécifiés. Il n'est pas indifférent que ce lecteur interpellé soit identifiable dan certains cas en tant qu'homme et, plus précisément, en tant que critique littéraire à qui est reprochée une lecture abusivement autobiographique des textes de Colette:

- «Homme, mon ami, tu plaisantes volontiers les oeuvres, fatalement autobiographiques, de la femme.» (316)

17 Derrière une telle formule, et ses procédés de généralisation, il est en effet difficile de ne pas voir une allusion relativement transparente à ce que sont les discours critiques en vigueur à cette époque sur la littérature féminine dans le cadre de laquelle s'est opérée la 
reconnaissance progressive de l'oeuvre de Colette. Se lisent ici et l'écho du mépris relatif dans lequel est tenue la catégorie minorée de la littérature féminine et, grâce au jeu subtil de la ponctuation - «fatalement autobiographique » est entre virgules ce qui suffit à caractériser l'autobiographie en tant qu'effet de lecture -, l'écho de la propension de la critique à voir dans la production féminine une production nécessairement autobiographique qui sera lue comme telle dans une logique qui en renforce la dévalorisation. Ce sont donc bien les a priori des lecteurs surtout masculins et les a priori de lecture dont sont victimes les ouvrages féminins, leur enfermement dans un horizon d'attente réducteur, qui sont dénoncés dans et par le texte.

D'autres figures de "mauvais » lecteurs hantent également l'oeuvre, toujours en liaison avec une lecture abusivement autobiographique, celle qui, en dépit de la distance temporelle et fictionnelle, conduit, par exemple, à confondre obstinément Colette et Claudine :

«Il y a encore des jeunes filles - trop jeunes pour prendre garde aux dates des éditions - qui m'écrivent qu'elles ont lu les Claudine en cachette, qu'elles attendent ma réponse à la poste restante... à moins qu'elle me donnent rendez-vous dans un "thé". Elles me voient peut-être en sarrau d'écolière, qui sait? en chaussettes?» (316)

19 Entre ironie - vis-à-vis des lectrices évoquées - et autodérision - dans le portrait qu'elle fait d'elle-même en sarrau et chaussettes -, ce passage joue à plusieurs niveaux : en se moquant de ce type de lectrice, Colette construit un lecteur virtuel qui s'en démarque par l'âge, les compétences lectorales et les motivations. Elle prend ses distances par rapport à la production littéraire de ses débuts tout en s'attirant les bonnes grâces d'un lecteur dont elle se fait un complice à travers une moquerie qui s'exerce contre des lectrices inexpérimentées qui en sont restées à confondre fiction et biographie au point de proposer des rendez-vous amoureux à l'auteur.

On notera pour finir que le personnage fictif de Vial constitue, lui aussi, une figure de mauvais lecteur dans la mesure où il fait, à sa manière, une lecture autobiographique de l'oeuvre de Colette, cherchant à mieux la connaître en utilisant ses oeuvres :

«A combien d'hommes dans votre vie avez-vous dit une chose pareille? Peut-être ne l'avez-vous dit qu'à moi? D'ailleurs je n'en trouve trace dans aucun de vos livres... aucun, non... Ce que vous venez de dire là se sépare du mépris de l'amour qu'on devine toujours, un peu, dans votre amour de l'amour... » (341, c'est Vial qui parle)

21 Présenté à travers les propos qui lui sont prêtés comme un lecteur assidu et compétent - jusqu'à un certain pédantisme - de l'oeuvre de Colette ${ }^{8}$, Vial est condamné pour cette posture abusive qu'il partage avec les autres lecteurs de Colette et que la narratrice récuse dans le commentaire dont elle fait suivre ses paroles :

«Je ne puis dissimuler le découragement jaloux, l'injuste hostilité qui s'emparent de moi quand je comprends qu'on me cherche toute vive entre les pages de mes romans ». (Ibid.).

Constituant à bien des égards une phrase-clé de l'oeuvre, cette formule inscrit dans le texte même, par le biais de la figure de Colette-écrivain, le rejet de la lecture autobiographique de ses oeuvres et a, pour le lecteur, valeur et de mise en garde et de mise en demeure de renoncer à ce type de lecture.

Il va donc de soi que les figures de mauvais lecteurs construites par le texte ne se confondent en rien avec le lecteur virtuel qu'il suppose. Au contraire, le lecteur virtuel 
- et éventuellement le lecteur réel - ne peut qu'être incité à se démarquer de ces mauvais lecteurs inscrits dans le texte comme repoussoirs et à occuper une autre position plus adéquate.

Cette position, le texte la dessine en creux en même temps qu'il construit pour la narratrice-auteur une position elle aussi nouvelle que le texte se fait fort de conquérir. Ce double déplacement s'opère à travers une série de procédures textuelles qui, complexifiant la texture même du texte, interdit au lecteur de venir réoccuper la position récusée de la lecture autobiographique. Il s'agit en fait de desserrer l'étau de ladite lecture et de créer du jeu entre autobiographie et fiction, c'est à dire un espace qui permette à l'écrivain de mener à bien le projet qui est le sien dans La Naissance du jour en l'imposant à son lecteur ou, du moins à ce lecteur souhaité qui n'est, sans doute, que l'envers du lecteur supposé qu'il s'agit de transformer.

L'épigraphe, là encore, livre certains éléments, en particulier dans sa seconde partie: «Patience, c'est seulement mon modèle ». Après la suspicion vis-à-vis d'un lecteur susceptible d'une erreur de lecture, vient un conseil («Patience») et une indication portant sur ce que doit être une lecture adéquate. Énigmatique, la formule s'éclaire progressivement dans le début du livre avant d'être partiellement explicitée lors de sa reprise au milieu du texte. Méditant sur des fragments de lettres retrouvées de sa mère, la narratrice-Colette s'essaie, à l'aube d'une vieillesse qui s'approche, à l'exercice de l'abstention dont les lettres maternelles lui fournissent le modèle, la Naissance du jour étant elle-même le récit d'un renoncement amoureux vis-à-vis du personnage de Vial.

Loin d'être le portrait de ce que Colette est effectivement, le texte construit donc le modèle de ce qu'elle voudrait devenir et d'une attitude qu'elle voudrait rejoindre mais qu'elle n'a pas encore rejointe et qui suppose une sorte d'exercice spirituel, un apprentissage progressif dont le texte est censé rendre compte. Parlant du moment où une femme commence à vieillir Colette écrit :

« (...)un âge vient où il n'est plus donné à une femme que de s'enrichir.

Elle entasse, elle recense jusqu'aux coups, jusqu'aux cicatrices (...) elle pèse, malgré elle, la valeur des dons. Elle les range peu à peu harmonieusement. Le temps et leur nombre fait qu'elle est obligée, dans la mesure où son trésor s'accroît, de se reculer un peu de lui, comme un peintre de son oeuvre. Elle recule, et revient, et recule, repousse à son rang quelque scandaleux détail, attire au jour un souvenir noyé d'ombre. Elle devient - par un art inespéré - équitable... Imagine-t-on à me lire, que je fais mon portrait? Patience, c'est seulement mon modèle."

27 Jouant de la polysémie du mot "modèle", l'écrivain répète ici à son lecteur l'avertissement de l'épigraphe (l'image de Colette que donne l'oeuvre n'est ni vraie ni ressemblante; elle ne doit pas être confondue avec la réalité présente; elle n'est que le dessein d'une figure à venir, à construire dans une écriture qui se veut prospective) tout en soulignant comment cette sagesse à conquérir est aussi, comme pour le peintre, une (bonne) distance à trouver par rapport à son objet, bonne distance qui permet de mieux le voir.

Ce que Colette propose à son lecteur - ou exige de son lecteur - c'est donc un retournement complet d'attitude, l'acceptation d'un changement total de grille de lecture par rapport à la lecture autobiographique habituellement pratiquée : au lieu de lire le texte en y voyant une Colette exposant aux yeux de tous ce qu'elle est dans la réalité, il s'agit de le lire comme l'instrument d'une démarche où elle s'efforce de dessiner ce vers quoi elle veut tendre afin de tenter de le rejoindre, où le texte devient le lieu de l'élaboration d'une fiction du moi qui, comme le soulignait la première épigraphe, n'est 
pas le moi. On notera que dans ces conditions le cadre familier et rassurant d'une pseudoautobiographie proposé au lecteur ne l'est que pour mieux le déstabiliser par la mise en place d'une logique autofictionnelle qui l'oblige à changer de posture lectorale pour adopter une position inédite, dictée par la narratrice à travers la construction d'une fiction de soi et d'une démarche prospective.

" Gribiche » ou la manipulation du lecteur

Le texte de "Gribiche » offre une autre variante en matière de relations entre Colette et son lecteur puisque l'écrivain y joue vis à vis de ce dernier une sorte de double jeu entre lien de confiance et effet de surprise : si l'enjeu est le même - amener le lecteur à une transformation radicale de sa position de lecture -, les modalités en sont plus complexes encore dans la mesure où, dans ce cas, le texte s'écrit à la fois avec et contre son lecteur.

Comme dans La Naissance du jour, "Gribiche» propose à son lecteur un univers apparemment familier et aisément reconnaissable dans la mesure où il s'inscrit dans la continuité explicite de textes antérieurs, construisant ainsi - tout en le présupposant - la figure d'un lecteur familier de l'univers colettien à qui de nombreux signes de reconnaissance sont largement proposés comme autant de balises. Censé se dérouler dans les années où Colette jouait au music-hall et raconter un épisode passé jusque-là sous silence - l'avortement de Gribiche et sa mort -, le récit s'inscrit, en effet, dans un cadre pseudo-biographique approximativement crédible pour le lecteur ${ }^{9}$ et dans le contexte intertextuel d'oeuvres comme La Vagabonde ou L'Envers du music-hall.

31 Il serait inutile et fastidieux d'énumérer ici les nombreuses références et allusions établissant ce lien intertextuel. Signalons seulement comment l'incipit du texte crée une impression de familiarité et de continuité :

«Je n'arrivais pas avant neuf heures quinze. A cette heure-là, la température et l'odeur du sous-sol avaient pris déjà toute leur force. Je ne désignerai pas d'une façon plus précise le music-hall où je jouais, entre 1905 et 1910, un petit rôle dans une revue. »(1145)

Outre un commencement in medias res sous-tendu par l'usage de l'imparfait, cet effet de familiarité provient de l'implicite contenu dans les deux premières phrases (arriver où? dans quel sous-sol?) mais aussi de la présence d'un « je » que les termes de « music-hall » et de « revue » rendent aisément reconnaissable au lecteur. Le texte impose en fait l'idée que se continue ou se renoue un dialogue seulement interrompu.

En même temps, Colette propose à son lecteur un pacte de lecture clairement déterminé par le fait que la nouvelle se donne comme projet de réécriture ou de rectification des textes antérieurs. Ce projet de réécriture s'énonce à travers deux formules qui se suivent à peu de distance dans les premières pages de la nouvelle. La première est une question dont le destinataire est largement indécidable puisqu'il peut s'agir de la narratrice ellemême qui, par scrupule, s'interroge sur sa démarche antérieure ou le lecteur supposé à qui elle prête ce qui pourrait être un reproche à elle adressé :

«Ai-je été, à cette époque, trop sensible à la convention de travail, de parade, de lumière, de vide cérébral, de ponctualité, de probité roide, qui régit le music-hall? M'a-t-elle inspiré de le dépeindre dans mainte page, avec un amour vif et superficiel et ce qu'il entraîne de banale poésie? Peut-être. » (1148)

Il est à noter que, si la première question met en cause la seule lucidité du personnage, la seconde implique clairement l'écrivain de sorte que le lecteur s'y trouve lui aussi plus directement impliqué à travers le jugement de valeur qu'elle suggère sur l'œuvre passée. 

directe à son égard :

Quelques lignes plus loin, une formule, cette fois assertive, condamnant la vision antérieure, vient confirmer la nécessité de la réécriture/rectification :

«Tout n'y était pas joie, tout n'y était pas si pur que je l'ai écrit. »(1149)

Parlant en tant qu'auteur, la narratrice propose donc à son lecteur une nouvelle dont la fonction proclamée est de venir rectifier/réécrire les textes antérieurs de l'écrivain, «Gribiche » se donnant (si l'on tient compte du contexte de publication ) comme la réécriture de La Vagabonde et de L'Envers du music-hall par l'auteur de Mes Apprentissages et de Ces Plaisirs... ${ }^{10}$ Supposé familier, comme on l'a vu, de l'univers colettien, le lecteur impliqué par le texte est également supposé ouvert à cette démarche de rectification qu'il est peut-être même réputé attendre ou souhaiter dans la mesure où elle est, à cette date, relativement familière à l'écrivain. A ce lecteur dessiné comme largement bienveillant et coopératif - contrairement ici à La Naissance du jour -, le texte s'emploie également à fournir, dans les pages qui suivent, les gages textuels ${ }^{11}$ de la réécriture annoncée. De ce fait - et c'est cela qui nous importe - il établit et accrédite une relation de confiance entre la narratrice et son lecteur, le texte s'écrivant - du moins en apparence - pour ou avec son lecteur plutôt que contre lui.

Qui plus est, dans le cadre de ce processus de réécriture, le texte modifie, par rapport aux textes antérieurs ${ }^{12}$, le rapport entre la narratrice, son objet et son lecteur. Disons, pour aller vite, qu'il infléchit le rapport que la narratrice entretient avec l'univers du musichall vers une plus grande extériorité ${ }^{13}$ et rapproche inversement cette dernière de son lecteur à travers le regard qu'elle porte sur un univers censé être largement étranger à ce dernier. Occupant ainsi une position de médiatrice entre l'univers représenté et le lecteur, la narratrice l'invite en même temps à une démarche pour laquelle elle accepte de le guider : son propre itinéraire au fil du récit - et qui consiste en la découverte progressive et la compréhension (dans les deux sens du mot) du sort de Gribiche - dessine pour le lecteur une voie vers une position que le texte lui propose et qu'il est supposé pouvoir accepter même si c'est au prix d'un effort à consentir, sur le modèle justement de celui que fournit la narratrice elle-même.

Cette position proposée au lecteur est suggérée à travers l'une des phrases de la nouvelle où l'emploi d'un pronom de la première personne du pluriel constitue une sollicitation

« (...) je sentis que la solidarité nous est plus facile que la sympathie » (1161).

Recourant à un présent, elle établit un constat qui lie narratrice et lecteur dans une même attitude vis-à-vis des personnages tiers et les situe tous deux entre une solidarité relativement spontanée - dont témoigne l'attitude du personnage - et une sympathie vers laquelle il est plus difficile de tendre compte-tenu de ce qui sépare, y compris socialement, la narratrice de ses camarades. Reconnue comme difficile, cette sympathie n'en est pas moins évoquée comme un effort à fournir que la narratrice sollicite implicitement d'elle-même mais également d'un lecteur supposé capable lui aussi de le fournir et donc de considérer sans condamnation hâtive, étant donné sa bonne volonté et sa relative largeur d'esprit, le secret de Gribiche.

Qui plus est, la narratrice montre la voie au lecteur en mettant en récit l'effort même qu'il importe d'accomplir pour accéder à cette position; il s'agit, pour elle, de renoncer à sa vision antérieure du music-hall dont elle dénonce « la banale poésie ». Ainsi au moment où se révèle au personnage le secret de Gribiche, elle exprime sa nostalgie face à ce qu'elle est en train d'abandonner malgré elle : 
"Je fermais les yeux pour mieux écouter des paroles qui me rouvraient un pays écarté de toute vérité, et même de toute vraisemblance. Paysage resplendissant, féerique bureaucratie (...). J'oubliais le lieu, le motif qui m'y avait entraînée... (...) Mais oui, mais certainement. Encore, encore!... Comme je les aime ainsi, sans défense contre les fables lorsqu'elles quittent leurs dehors d'employées économes et dures... Oublions tout, sauf l'extravagant, oublions même cette petite réalité martyrisée, couchée à plat sur son lit, devant une fenêtre à barreaux... »(1169).

On se bornera à commenter ici l'effet produit par l'utilisation de la forme impérative « oublions »: l'exhortation en forme de tentation que le personnage s'adresse à lui-même se généralise en un conseil du même ordre englobant le lecteur, mais de sorte que ce dernier se trouve par là-même interdit de pratiquer cette fuite vers la féerie, la fable et l'extravagant qui, un moment, tente le personnage. Intégrée dans le texte même, cette position y est donc dessinée à la fois comme tentation et impossibilité d'un retour en arrière, marquant ainsi le déplacement de la position de l'écrivain opéré par et dans le texte en même temps que la nécessité pour le lecteur d'accompagner, encore une fois, ce changement de position.

Par nombre d'aspects, le texte de «Gribiche » tend donc à instaurer et à maintenir une relation de confiance entre auteur et lecteur. Pourtant, le texte réserve, en même temps, bien des surprises à ce dernier dans la mesure où le texte s'applique à saper insidieusement les fondements mêmes sur lesquels il prétend être bâti et à emmener le lecteur loin de l'endroit où il semblait le conduire. C'est que la narratrice-auteur n'y occupe pas réellement la position qu'elle déclare adopter quand elle annonce son projet de réécriture. Le texte de « Gribiche » ment sur ce qu'il fait, et ce parce que l'auteur y joue double jeu avec son lecteur. Feignant d'écrire pour lui et avec lui, elle écrit aussi contre lui en décidant de n'être pas vraiment là où elle dit qu'elle est et là où on la croit, confrontant par là son lecteur à l'obligation d'une nouvelle modification de sa position de lecture, modification qui cette fois est exigée sans être annoncée.

La nouvelle se donne, en effet, ostensiblement pour telle afin de mieux oublier de dire ce qu'elle est aussi, une réécriture des textes consacrés à l'enfance et au pays natal comme en témoignent diverse allusions émaillant la seconde partie de la nouvelle et deux formules plus explicites :

« je songeai au jardin de mes années sereines» (1162)

"Chez vous aussi çà sent bon... çà me rappelle... une odeur de mon pays » (1163).

Les mots « jardin » et « pays » suffisent, en effet, à convoquer tout un intertexte colettien auquel s'articule le texte de Gribiche puisqu'il apparaîtra bientôt dans la nouvelle que cette odeur qui participe en apparence de la poésie/nostalgie du pays natal est en réalité l'odeur même de l'absinthe abortive qui sera fatale à Gribiche de sorte que la nécessité ailleurs affirmée d'abandonner toute vision poétique facile du music-hall se révèle soudain pouvoir s'appliquer aussi aux textes de l'enfance.

Le lecteur se retrouve donc face à un autre texte que celui qu'on lui avait fait délibérément attendre, un texte qui le place soudain dans une position d'autant plus inconfortable qu'on lui donne à lire une autre réécriture que celle qui lui avait été annoncée et que cette réécriture le confronte sans prévenir avec le monstrueux que représente la figure oxymorique de la mère avorteuse.

En même temps, de nouvelles exigences se font jour, de la part de l'auteur, quant à la position que le lecteur se doit d'adopter face à ce monstrueux qui lui est donné à voir. 
Pour en juger, il convient, ici encore, de prendre en compte une des formules du texte qui utilise un "nous » pour englober dans le même énoncé la narratrice et son lecteur :

«Je n'avais jamais vu d'accidents graves au music-hall (...) J'avais comme tout le monde imaginé leur fin avec cette complaisance obscure et vague que nous ressentons pour tout ce qui nous fait horreur » (1158).

Correspondant à une remise en question de l'idée que le personnage se fait des risques mortels encourus par les artistes, ce passage est un écho de la fin d'un des textes de L'Envers du music-hall dans lequel la narratrice y évoque ce qu'elle appelle « une âme de spectateur ", " une âme(...) tremblante, avide d'horreur et pusillanime» (Pl.2, 261). Or cette attirance horrifiée n'est rappelée dans « Gribiche » que pour être aussitôt déplacée d'un objet factice (la mort accidentelle et spectaculaire lors d'un exercice dangereux) propre à attirer les regards de qui s'installe dans la position de spectateur, vers un autre objet (Gribiche et son secret). La mort encourue revient donc mais sur un tout autre mode que celui initialement imaginé; elle revient sur un mode mineur, sous une forme en apparence banale et quotidienne mais dans lesquels les pouvoirs de l'horreur ${ }^{14}$ ne sont pas moins présents. Ce sont eux que Colette donne à voir à son lecteur mais en exigeant expressément de lui qu'il abandonne toute idée du spectaculaire dans l'horreur.

Amené là où il ne s'y attendait pas - la confrontation avec le monstrueux - le lecteur se voit donc sommé en outre de réviser ses conceptions et de modifier sa position de lecture: loin de toute complaisance fascinée et facile vis-à-vis du spectaculaire, il est conduit par l'auteur à le percevoir dans ce qu'il a de quotidiennement invisible et à apprendre à l'approcher d'une autre façon puisque ce refus du spectaculaire se traduit aussi dans l'écriture colettienne par une exigence de retenue qu'illustrent les choix narratifs de l'écrivain ${ }^{15}$ et qu'affirme dans le texte lui-même une remarque de la narratrice constatant que ses camarades «exprimaient le monstrueux en termes modérés » (1172).

On voit donc comment Colette, écrivant aussi contre son lecteur, l'amène sur des terrains où il n'avait pas nécessairement prévu d'aller, trompé qu'il était par l'horizon d'attente en partie fallacieux proposé par le pacte initial de réécriture. La relation de confiance qui semble s'établir dans le texte est en même temps un moyen textuel de le "déranger " pour le conduire là où il n'est pas averti qu'il doit aller. Ecriture contre et écriture avec (le lecteur) se mêlent donc dans un texte qui a, une fois encore, pour objectif un déplacement concomitant de la position énonciatrice et de la position de réception. Réécriture donc comme la narratrice-auteur l'annonçait mais réécriture qui ouvre sur autre chose que ce qu'elle annonçait, obligeant ainsi le lecteur à un déplacement qui n'est pas seulement celui qui lui était annoncé et qu'il était prêt à accepter. D'où le malaise qui sourd pour lui à la lecture de la nouvelle et la remise en question finale où le conduit, d'une main de fer, la narratrice-auteur en lui donnant à voir une horreur dépouillée des oripeaux faciles du spectaculaire.

« La Lune de pluie » ou le feuilleté des lectures

50 «La Lune de pluie» offre un autre exemple encore de cette capacité de Colette à renégocier, au sein même de ses textes, les modalités de leur lecture en amenant son lecteur à déplacer, modifier sa position de lecture. Chronologiquement le dernier des trois textes examinés ici, il offre en la matière une démarche qui parait à la fois plus sûre d'elle même et plus apaisée. La narratrice y accompagne en effet son lecteur plus qu'elle ne le critique (comme dans la Naissance du jour) ou ne cherche à le surprendre (comme 
dans "Gribiche ») et ce, en lui proposant plusieurs modèles possibles d'écriture et de lecture d'une seule et même histoire.

51 Le premier de ces modèles est le modèle romanesque. La narratrice évoque d'ailleurs, non sans quelque autodérision volontaire, la tentation du romanesque qui naît en elle avec l'histoire Barberet et plus généralement quand elle cède aux divagations - passées mais aussi présentes - de son imagination ${ }^{16}$. Or cette potentialité romanesque de l'histoire Barberet trouve à s'actualiser au sein de la nouvelle dans l'embryon de récit que la narratrice propose à sa couturière, Marie Mallier. A travers la réaction de ce personnage, Colette construit une figure possible de lecteur et donne ainsi à voir, en même temps, un modèle de lecture qui, en fonction de son horizon d'attente spécifique, permet d'interpréter l'histoire racontée comme le « commencement » (89) d'un récit romanesque impliquant lui-même un prolongement qui réponde à des modalités narratives prévisibles et codifiées. Et d'une certaine façon, ce prolongement potentiel est lui-même donné, au moins virtuellement, à travers l'évocation d'un feuilleton que justement la narratrice tente d'écrire et ne parvient pas à écrire " prenant en dégoût (son) feuilleton chaque fois qu'(elle) tentai $(\mathrm{t})$ d'y introduire le "mouvement", l'aventure rapide et un brin de terreur ». (85). On le retrouve aussi, toujours virtuellement, à travers la référence à Fantomas que le mari d'Adèle conseille à Rosita de lire quand celle-ci tente de le prévenir des machinations tramées par sa soeur (119). Bref, le texte de la nouvelle suggère donc tout en la refusant ce que pourrait être, tant du point de vue de son écriture que de sa lecture, la version feuilletonesque de l'histoire qu'il raconte.

52 On signalera d'ailleurs que la fin même de la «La Lune de pluie » qui choisit de laisser le lecteur dans l'indécision concernant le deuil constaté d'Adèle livre, au moins comme une possibilité, un dénouement de l'histoire Barberet dans lequel l'efficience éventuelle des pratiques magiques de Délia introduit justement ce «brin de terreur» dont parle la narratrice, laissant ainsi ouvert jusqu'à la fin le modèle de la lecture romanesque présent dans le texte.

Un deuxième mode de rapport possible à l'histoire Barberet soulève, quant à lui, la question - toujours épineuse, nous l'avons vu, quand il s'agit de Colette - d'une écriture et d'une lecture autobiographiques des textes. Comme précédemment, ce modèle est présent à travers la tentation d'identification éprouvée par la narratrice elle-même vis-àvis de ce double potentiel qu'est Adèle, tentation de l'identification qui, toute efficace qu'elle puisse être au moment de la rencontre avec les soeurs Barberet de par ses vertus cathartiques, n'en est pas moins mise à distance par la narratrice quand elle s'interroge :

"Que faisais-je, dans un lieu qui m'eût dû être interdit, auprès d'une femme assez jeune pour que rien ne marquât sa condition d'épouse, et qui ne manifestait ni vertus, ni élévation, ni même autant d'intelligence qu'un animal? Il s'agit, j'y insiste, d'une période de ma vie à laquelle la maternité et l'amour heureux n'avaient pas encore fourni leur merveilleux lieu commun » (99).

Lisible au départ comme une question que semble se poser la narratrice à son époque Barberet, la première phrase se transforme, en effet, à travers le commentaire que constitue la seconde, en question que se pose la narratrice plus âgée et clairement soucieuse de s'adresser directement à son lecteur pour mieux marquer les distances qui la séparent de ce qu'elle fut autrefois.

55 Vécue comme elle l'est au départ sur le mode de l'identification, l'histoire Barberet s'avère, en effet, n'être pas racontable à Sido, non plus d'ailleurs qu'elle n'est racontable à Annie de Pène, l'amie de la narratrice: la raconter à sa mère reviendrait pour la 
narratrice à dévoiler des secrets de sa vie privée qu'elle veut lui cacher (107); la raconter à Annie de Pène l'exposerait à la réprobation de son amie, réprobation suscitée par une certaine complaisance sentimentale de la narratrice vis-à-vis d'elle même. Celle-ci met donc en scène dans le texte de la nouvelle son propre renoncement à l'idée de livrer un récit qui, confidence transparente sur sa propre vie et ses états d'âme, serait trop aisément lisible par ses destinataires sur le mode autobiographique d'une confession à peine déguisée. A travers le refus d'une mise en récit où prédominerait trop visiblement l'épanchement personnel, la narratrice met donc à distance, comme dans la Naissance du jour, le modèle même de lecture autobiographique qui a si souvent prévalu à propos de ses oeuvres. Elle met en scène un modèle d'écriture et un modèle de lecture pour mieux les récuser ensemble, préférant raconter à Marie Mallier une autre version de l'histoire Barberet qui coïncide, on l'a dit, avec le modèle romanesque.

On notera toutefois que, comme pour le feuilleton virtuel ou l'allusion au récit destiné à Marie Mallier, le texte de la nouvelle livre néanmoins au lecteur un court échantillon de ce qu'aurait pu être le récit fait à Annie de Pène si la narratrice n'y avait pas justement renoncé :

«Cette fenêtre, Annie, où, comme je fis autrefois, une jeune femme délaissée passe tout son temps à attendre, à écouter... »(89).

Elle procéde ainsi par ébauche d'un récit potentiel que le texte de La Lune de pluie donne et refuse en même temps à son lecteur et que la narratrice livre tout en s'en démarquant par la manière dont elle accentue volontairement la tonalité sentimentale du fragment proposé.

S'il en est ainsi, c'est qu'à côté de ces deux modalités romanesque et autobiographique, le texte de La Lune de pluie s'emploie lui aussi à construire encore une autre position de la narratrice par rapport au récit et symétriquement une autre position pour son lecteur. Devenant, à la fin de la nouvelle, auditrice de l'histoire que lui raconte Rosita, elle se met elle-même en scène dans la position d'une ethnographe à la fois fascinée et incrédule face aux croyances et aux pratiques magiques des deux soeurs. Ce point de vue se caractérise par une position à la fois de proximité compréhensive et d'extériorité proche de celle déjà repérée dans "Gribiche » : la proximité compréhensive s'exprime à travers la confiance particulière manifestée par Rosita et la main que lui tend la narratrice (118); l'incrédulité par ses questions empreintes de scepticisme et ses commentaires où s'exprime son malaise, questions et commentaires construisant ensemble une position volontairement proche de celle qui est supposée être celle du lecteur de la nouvelle et qu'actualise, par exemple, cette fausse interrogation à lui adressée :

« Des chuchotements, une foi obtuse, et même une habitude du quartier, étaient-ce là les forces, les philtres qui procurent l'amour, décident de la mort et de la vie, déplacent cette altière montagne : un coeur indifférent? »(120)

Ce même point de vue ethnographique se caractérise également par le fait que le texte inscrit ces croyances et pratiques des soeurs Barberet dans un ensemble plus vaste de pratiques féminines et populaires dont la narratrice signale qu'il n'est connu de personne parce que n'accédant jamais à la publicité d'une publication :

«Ce qui n'est écrit nulle part, sauf par des mains maladroites sur des cahiers d'écoliers, ou sur du papier quadrillé de gris, mince, coupé aux plis, jauni aux bords, cousu de coton rouge; ce que la sorcière légua au rebouteux, que le rebouteux vendit à l'obsédée d'amour, que l'obsédée céda à une autre maudite; ce que la crédulité et la mémoire souillée d'une fille pure peuvent recueillir dans les antres qu'une ville insondable ménage entre un cinéma neuf et un bar-express, je 
l'entendis, transmis, vanté à Rosita Barberet par des veuves victorieuses, de lubriques épouses, des fiancées délaissées et attentatoires, la rêverie effrénée des femmes seules... » (Ibid).

60 Colette en tant qu'écrivain se donne donc, à travers la figure de la narratrice de la nouvelle, comme celle qui rend compte de ce continent noir des croyances et pratiques féminines qu'on ne trouve que dans ces documents modestes, largement illégitimes et occultés que sont cahiers d'écolier et supports réalisés à partir de mauvais papier ${ }^{17}$. Elle construit par là une position d'écrivain ethnographe jouant le rôle de médiateur $-\mathrm{y}$ compris social - entre ses personnages et ses lecteurs inscrits dans le texte. Elle désigne en même temps à ces lecteurs une position de lecture qui réponde à ce modèle d'écriture par elle adopté. Au déplacement de son propre point de vue sur l'histoire Barberet est censé répondre le même déplacement du lecteur au delà d'une lecture purement romanesque ou identificatoire de l'histoire qui lui est rapportée.

61 Par la démultiplication prismatique de l'histoire Barberet, Colette fait donc coexister, dans La Lune de pluie, plusieurs modèles d'écriture et de lecture, plusieurs positions pour le narrateur et le lecteur. En mettant en scène sa propre évolution par rapport à ces divers modèles possibles, elle joue vis à vis de son lecteur un rôle quasi pédagogique puisqu'elle le guide vers la tierce position vers laquelle elle l'invite à se déplacer : alors que les modèles romanesque et autobiographique de traitement de l'histoire Barberet sont suggérés mais laissés inaccomplis, le modèle ethnographique est, pour sa part, clairement revendiqué et proposé comme tel à un lecteur lui-même sollicité d'y répondre en adoptant le mode de lecture qui lui est prescrit.

62 Mais, en même temps, ce qui fait l'originalité et la souplesse du dispositif mis en place par Colette dans cette nouvelle, c'est sa capacité à faire coexister ces différents modèles d'écriture et de lecture, certes en les hiérarchisant, mais sans pour autant les récuser. $\mathrm{Si}$ le dernier modèle est, en effet, clairement donné par le texte comme celui que revendique l'écrivain, les autres modalités - qui sont suggérées et mises à distance, mises à distance mais partiellement livrées - ne font jamais l'objet d'un total rejet ni d'une complète condamnation, ne serait-ce que parce qu'elles sont prises en charge par la narratrice ellemême comme un moment de sa propre trajectoire. De cette façon, la nouvelle ménage, en quelque sorte, suffisamment d'espace pour que son lecteur potentiel puisse la lire selon les différents modèles proposés comme si Colette guidait désormais avec plus de souplesse et de tolérance son lecteur vers la position qu'elle souhaite lui voir adopter, peut-être parce que sa reconnaissance en tant qu'écrivain est à cette date plus solidement établie.

63 Les trois oeuvres analysées présentent donc des variantes significatives d'une commune stratégie visant à renégocier et modifier le modèle de lecture qui leur est applicable, par rapport à un modèle que le texte suppose ou qu'il feint de proposer pour mieux le remettre en question à travers un jeu de déplacement imposé au lecteur.

64 Concernant les procédés mis en oeuvre, il est à noter qu'il n'y a pas dans les rapports de Colette et son lecteur de relations d'affrontements directs et frontaux. Le texte ménage, au moins en apparence, un lecteur dont on a vu qu'il était souvent proche de ce que Colette pouvait en connaître, en lui offrant des éléments qui lui permettent une reconnaissance de ce qui lui est familier et en ne rejetant jamais d'emblée les lectures habituellement faites de son oeuvre.

65 En revanche, lui est imposée à chaque fois une remise en question de ses modèles de lecture selon des modalités qui varient selon les textes et au fil du temps. Dans $L a$ 
Naissance du jour, la construction explicite de figures de «mauvais lecteurs » a pour finalité la renégociation d'un pacte de lecture à travers laquelle le lecteur est invité à renoncer à la lecture autobiographique des textes colettiens. Dans "Gribiche», la proximité partiellement illusoire de la narratrice et de son lecteur est au service d'une exigence de changement plus radical et plus dérangeant encore. Dans «La Lune de pluie ", l'invitation au déplacement et à l'adoption d'une nouvelle position de lecture est toujours présente mais sur un mode moins directif et plus serein qui laisse davantage de latitude au lecteur, peut-être parce que Colette y est désormais plus sûre d'elle-même et de ses moyens.

C'est que les enjeux de cette démarche réitérée vis à vis du lecteur s'éclairent d'être mis en perspective. Dans des oeuvres qui se plaisent à superposer personnage, narrateur et auteur en jouant sur l'ambiguïté générique, ce déplacement que le texte sollicite de son lecteur ou qu'il lui impose est intimement lié à la modification de la figure de l'écrivain que construit ce même texte et, par là-même, aux étapes d'une trajectoire littéraire orientée vers la recherche d'une plus grande légitimité : la position que Colette s'efforce de faire adopter à son lecteur tend à renforcer sa légitimité d'écrivain en tant que celle-ci dépend étroitement du modèle de lecture proposé par le texte.

Par là-même, se donne aussi à voir, à travers ces rapports qui se construisent entre Colette et son lecteur, la position de Colette dans le champ littéraire et la spécificité de sa trajectoire d'écrivain.Tous ses efforts de renégociation des contrats de lecture participent de la volonté, au fur et à mesure que sa reconnaissance se renforce, de passer d'une logique dominante du succès à une logique du prestige. Pour cela, elle ne cesse de s'employer à redéfinir les modalités de sa lecture et à retravailler sa figure d'écrivain. Mais, on notera qu'elle a toujours, en même temps, le souci de conserver son public antérieur, de lui laisser toujours la possibilité de trouver une place dans ses textes, ce qui témoigne à sa façon de la position médiane qu'elle tend à occuper dans le champ littéraire, dans une zone qui reste intermédiaire entre succès et prestige.

\section{NOTES}

1. Cet article est la version remaniée d'une conférence proposée dans le cadre du CNA en février 2002. Je voudrais dire ici ce qu'il doit à la discussion qui, faisant suite à mon exposé, a enrichi ma réflexion, en particulier, à travers la question du rôle pédagogique qui peut être celui de l'auteur.

2. Cette perspective est celle adoptée dans l'ouvrage (lui-même tirée de ma thèse) : Les mécanismes de classicisation d'un écrivain : le cas de Colette, Université de Metz, "Recherches textuelles $n^{\circ} 4 ", 2000$.

3. Il est à signaler qu'il existe une thèse qui traite, dans une perspective un peu différente, de ces rapports entre Colette et son lecteur et qui porte sur les débuts de l'écrivain : Anne Poskin, Colette et son lecteur (1900-1920), Université de Liège, 1999.

4. La Naissance du jour a paru en 1928; "Gribiche" en 1937 dans le recueil Bella-Vista; "La Lune de pluie" dans le recueil Chambre d'hôtel en 1940. Les deux premiers textes figurent 
dans le tome 3 de l'Edition de la Pléiade, le troisième dans le tome 4. Pour les citations, nous indiquerons entre parenthèses les références aux pages de cette édition.

5. Cf, G. Prince, "Introduction à l'étude du narrataire", Poétique n¹4, 1973, pp. 178-196.

6. Cf Gérard Genette, Seuils, Paris : Seuil, 1987, p. 146.

7. Par exemple : allusion à la table à écrire rapportée de Bretagne; évocation des bêtes familières qui l'entourent pendant qu'elle écrit et qui renvoie aussi bien aux textes antérieurs qu'à l'iconographie qui circule dès cette période; références plus ou moins transparentes à ses textes antérieurs.

8. Son commentaire est une référence directe à un passage de La Vagabonde.

9. Même si, en fait, il y a manipulation des dates (voir à ce sujet Pl. 3, 1853).

10. Ces plaisirs... (première version de Le Pur et l'Impur) a paru en 1932; Mes Apprentissages (lui-même réécriture de la période Willy) en 1936.

11. A travers tout une série d'effets de reprises et de décalages qu'il serait trop long de détailler ici.

12. Cela apparait si l'on confronte le texte de "Gribiche" aux analyses développées par A. Poskin (cf., supra, note 3) sur L' Envers du music-hall..

13. A travers, en particulier, une attitude d'autodérision concernant ses velléités de "carrière" et les rôles qu'on a pu lui faire tenir (voir Pl. 3, pp. 1149 et 1175).

14. Cette formule est, bien sûr, empruntée au titre de l'ouvrage de Julia Kristeva, Pouvoirs de l'horreur, Paris, Seuil, 1980.

15. Voir, à ce sujet Pl. $3,1857$.

16. Ne se dépeint-elle pas, en effet, quittant le domicile des deux soeurs en "emportant un romanesque butin de conjectures" : "La soeur malade - et pourquoi pas folle mélancolique? ou languissante d'un langoureux amour? Ou frappée de monstruosité et tenue dans l'ombre? Voilà comme je suis quand je me laisse aller"(84).

17. C'est là, notons-le au passage, une occasion de faire allusion aux Claudine tout en s'en démarquant - Colette n'est plus celle qui écrit dans des cahiers d'écolier comme Claudine mais celle qui rend compte de ce que d'autres écrivent dans ces mêmes cahiers - et de se situer aussi par rapport à des écrits forcément féminins puisqu'ils trouvent place sur des supports confectionnés par des mains couturières - elle n'est pas une femme qui écrit sur ces supports mais, là encore, celle qui transcrit ce que d'autres peuvent y écrire. 